\title{
Correlation Study between Telescope Array Lightning Location System and ground Surface particle Detector
}

\author{
Takeshi Okuda* \\ Ritsumeikan University \\ E-mail: budadicrr.u-tokyo.ac.jp
}

The Telescope Array (TA) experiment detects air-showers induced by ultra high energy cosmic rays. The TA ground Surface particle Detector array (TASD) observed some short-duration bursts of air-shower like events. These events were evidently correlated with lightning. Therefore, we have deployed detectors for lightning discharge in the vicinity of the TASD. We describe the performance of the Telescope Array Lightning Location System (TALLS) and report the correlation study between TALLS and TASD.

36th International Cosmic Ray Conference -ICRC2019-

July 24th - August 1st, 2019

Madison, WI, U.S.A.

\footnotetext{
* Speaker.
} 


\section{Introduction}

Some high energy radiation bursts were observed by the Telescope Array Surface Detector(TASD), and correlated with lightning discharge detected by National Lightning Detection Network [U] []. In order to investigate these relation between high energy radiation and identified lightning discharge with higher statistics, I constructed lightning location system for TASD.

Telescope Array is located in midwest in Utah, USA (Lat $39.3^{\circ} \mathrm{N}$, Lon $112.9^{\circ} \mathrm{W}$, Alt $1400 \mathrm{~m}$ ). It mainly consists of 3 atmospheric fluorescence telescope stations called FD and 507 ground surface particle detectors called SD, see Figure W(Left). TASD is dual layer plastic scintillation detector, whose detection area is $3 \mathrm{~m}^{2}$ for each, see Figure $\mathbb{W}$ (Right). The shower trigger judgement of TASD is 3 adjacent detectors which was hit by 3 or more particles in $8 \mu \mathrm{s}$. Once triggered, all waveforms, which correspond to more than 0.3 particle, in $\pm 32 \mu$ s from triggered time are collected from all detectors [B]].
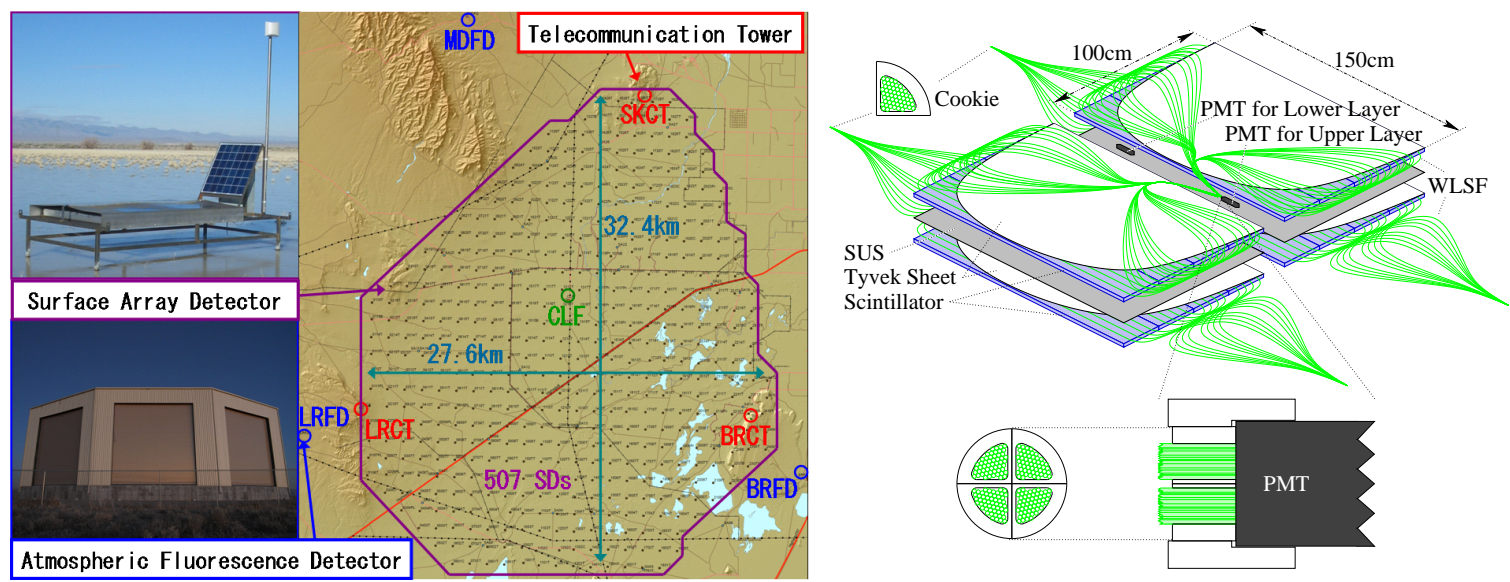

Figure 1: Telescope Array Experiment. Left: 507 ground surface particle detectors (tiny dots above) are surrounded by 3 atmospheric fluorescence telescope stations. Right: Particle detecting part of TASD consists of 2 layers of plastic scintillators. Each scintillation layer has $1.2 \mathrm{~cm}$ thickness and provides information respectively via wavelength-shifting fibers.

There are some precedent observations for the relation between high energy radiation and identified lightning discharge [䧃] [可] [圆]. The coverage area of precedent observation is several $\mathrm{km}^{2}$ at most, whereas TASD is deployed $680 \mathrm{~km}^{2}$. The radiation detector of precedent observations is mainly NaI. It can measure the radiation energy but the detection area is small and the response is slow. TASD is plastic scintillation detector. It cannot measure the radiation energy but the detection area is more than 100 times larger and the response is 10 times faster. Therefore, TASD can measure the statistical time profile of the high energy radiation, at different position in high energy air shower like phenomena by lightning. The precedent observations measure the energy of radiation and TASD measures spatial distribution of radiation. Therefore, TASD is unique and complementary to observe high energy radiation from lightning. TASD is running now and located where is stable lightning discharge in summer. In addition, TASD is the largest radiation detector with high sensitivity for electromagnetic component in the northern hemisphere. Near future, there 
is no promising plan to deploy radiation detectors in such a large area for radiation from lightning. Therefore, I tried to detect lightning discharge at Telescope Array site as many as possible.

\section{Lightning Location System}

Telescope Array Lightning Location System(TALLS) consists of 4 or 5 lightning detectors (Figure [2). In order to cover TASD, lightning detectors were deployed surrounding TASD. The first detector(LDA) was deployed in September 2014 at Cosmic Ray Center which is workshop for TA, in Delta City where the TA operators usually stay. The second and third detectors(LDB, LDC) were deployed in September 2015 at southern FD stations with solar battery system. The fourth detector(LDD) was deployed in March 2016 at northern FD station with solar battery system. The central data server was placed in Cosmic Ray Center and stored all data from local DAQ-PC beside lightning detector. The local DAQ-PC is controlled via this central data server remotely.
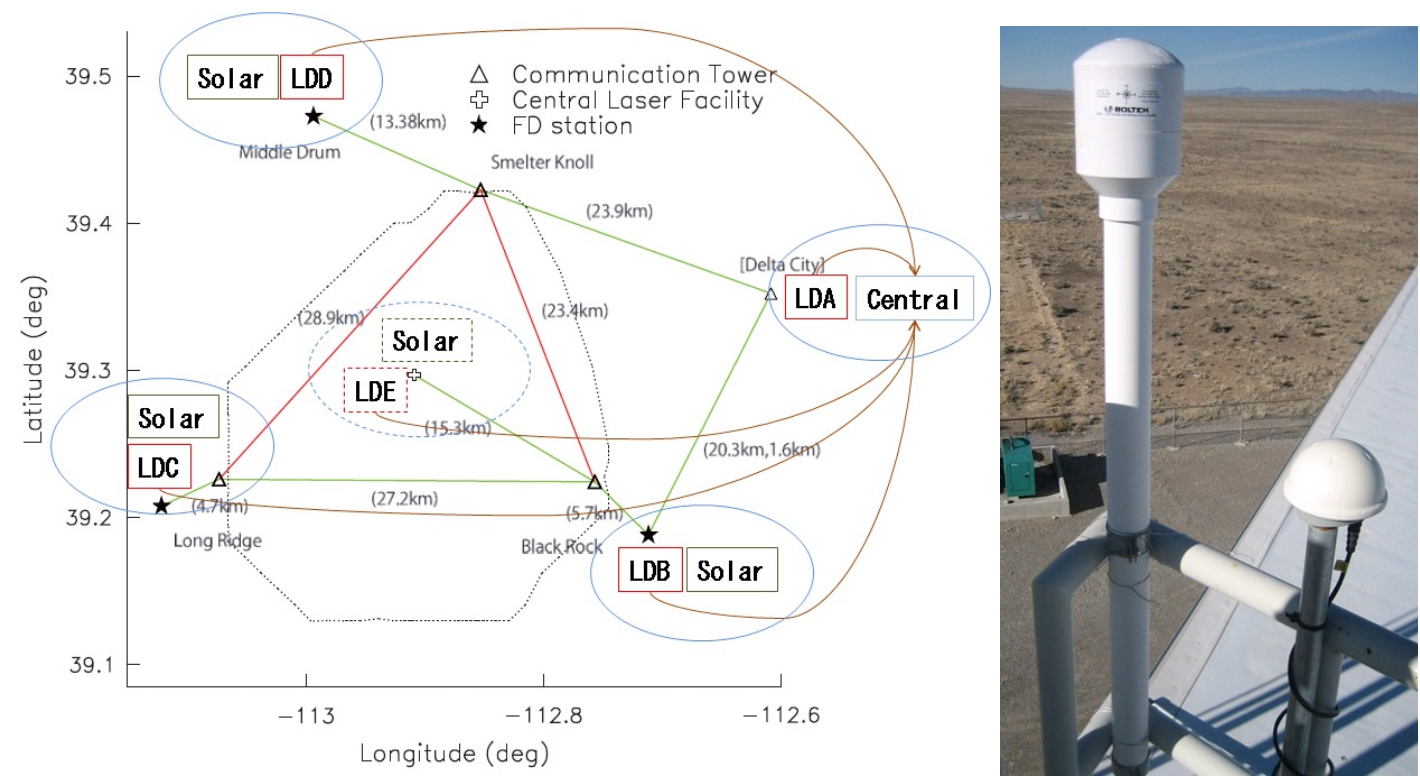

Figure 2: Lightning Location System Left: 4 lightning detectors are located surrounding TASD and running now. LD? shows detector name. Right: Antennas for lightning detector and its GPS on FD station.

The antenna for lightning detector consists of omnidirectional electric field antenna and orthogonal magnetic field loop antennas. Therefore, it outputs 3 waveforms at once. Each waveform has 512 data points for $51.2 \mu \mathrm{s}$. Two magnetic field loop antennas are orthogonal, therefore the ratio of the magnetic waveform amplitude indicates the direction of the lightning discharge from the detector. This method is called Magnetic Direction Finder(MDF). In principle, the lightning location by MDF can be determined by 2 detectors at minimum. However, this estimated direction has somewhat large error by the local environment around detector. Therefore, the accuracy of the estimated lightning location is not good. The other method uses the signal arrival timing for each detector. It is called Time Of Arrival(TOA). The time difference of a pair of detectors describe a hyperboloid as candidates of the lightning location. In principle, the lightning location by TOA 
can be determined by 4 detectors at minimum. This method requires the accurate timing of signal arrival for enough accuracy of the estimated lightning location. Therefore, GPS timing module is used to record the waveform timing. The locating accuracy is less than several hundred meters.

As written above, TOA needs 4 detectors to determine the lightning location uniquely at all times. However, TOA with 3 detectors can also determine the lightning location, but sometimes as double root. Even in this case, MDF can be used to choose the proper location from two candidates. Although MDF does not have good accuracy, it is enough to choose one of two. Therefore, TALLS use both methods to determine the location for 3-fold coincidence event. Especially, in even case of the malfunction of a detector, TALLS can keep the minimum locating ability.

\section{Observation and Correlation}

Observation status after fourth detector's deployment is shown in Figure B], for 994 days. Because the trigger threshold of the detector is set at low level, the trigger rate is more than the data output rate and then the number of alive detector decrease under the huge thunderstorm. Basically, these detectors run stably and the installed solar battery systems provide enough power even in winter.

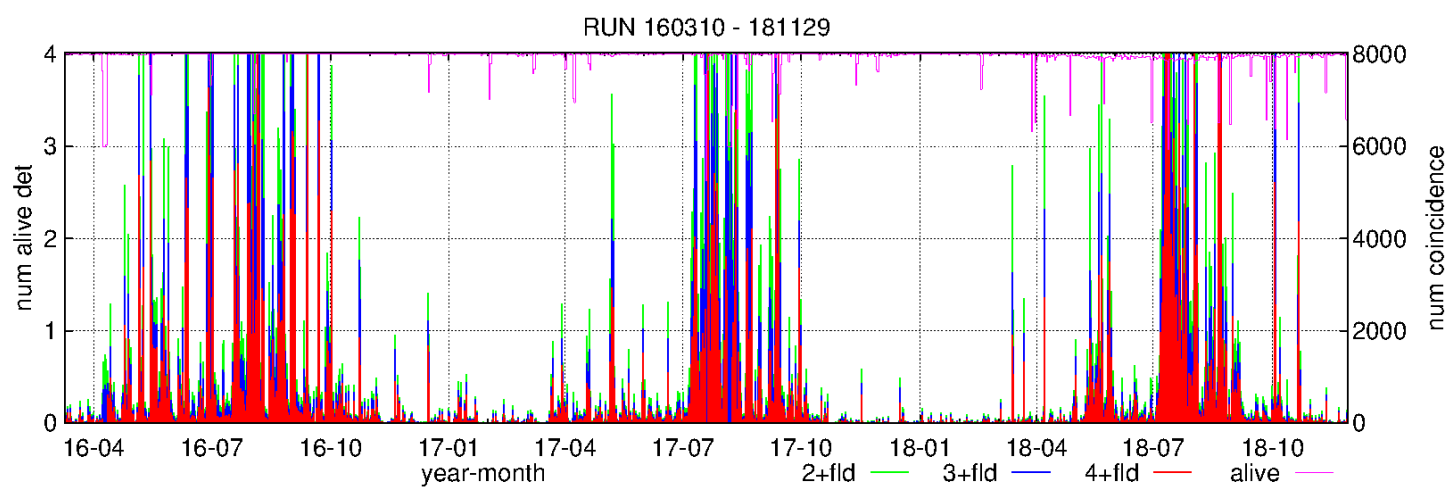

Figure 3: Running Status and the number of daily coincidence events after fourth detector was activated. Purple line shows the number of daily alive detector which is calculated in 1 minute resolution. Bars show the number of daily $n$-fold coincidence events. The width of coincidence is $400 \mu$ s. The maximum number of 4-fold coincidence in a day is more than 20000.

As an example, I show a 4-fold coincidence event taken on the afternoon of March 22 in 2016, 12 days after the deployment of the fourth detector. The electric field waveforms are almost same shape for 4 detectors whereas the magnetic field waveforms have various amplitude of east-west and north-south components for 4 detectors (Figure $\$$ ).

I checked the timing correlation between TASD and TALLS for 994 days, shown in Figure 5 . The definition of TASD burst is 3 or more events in $1 \mathrm{~ms}$. The burst event means the TASD event that consists of the burst. For TALLS data, I only use 3-fold coicidence timing. Although there are chance coincedince between TASD and TALLS, it is clear that the lightning discharge occurs around within $\pm 1 \mathrm{~ms}$ from air shower like event. In addition, there are subsequent discharge, not 

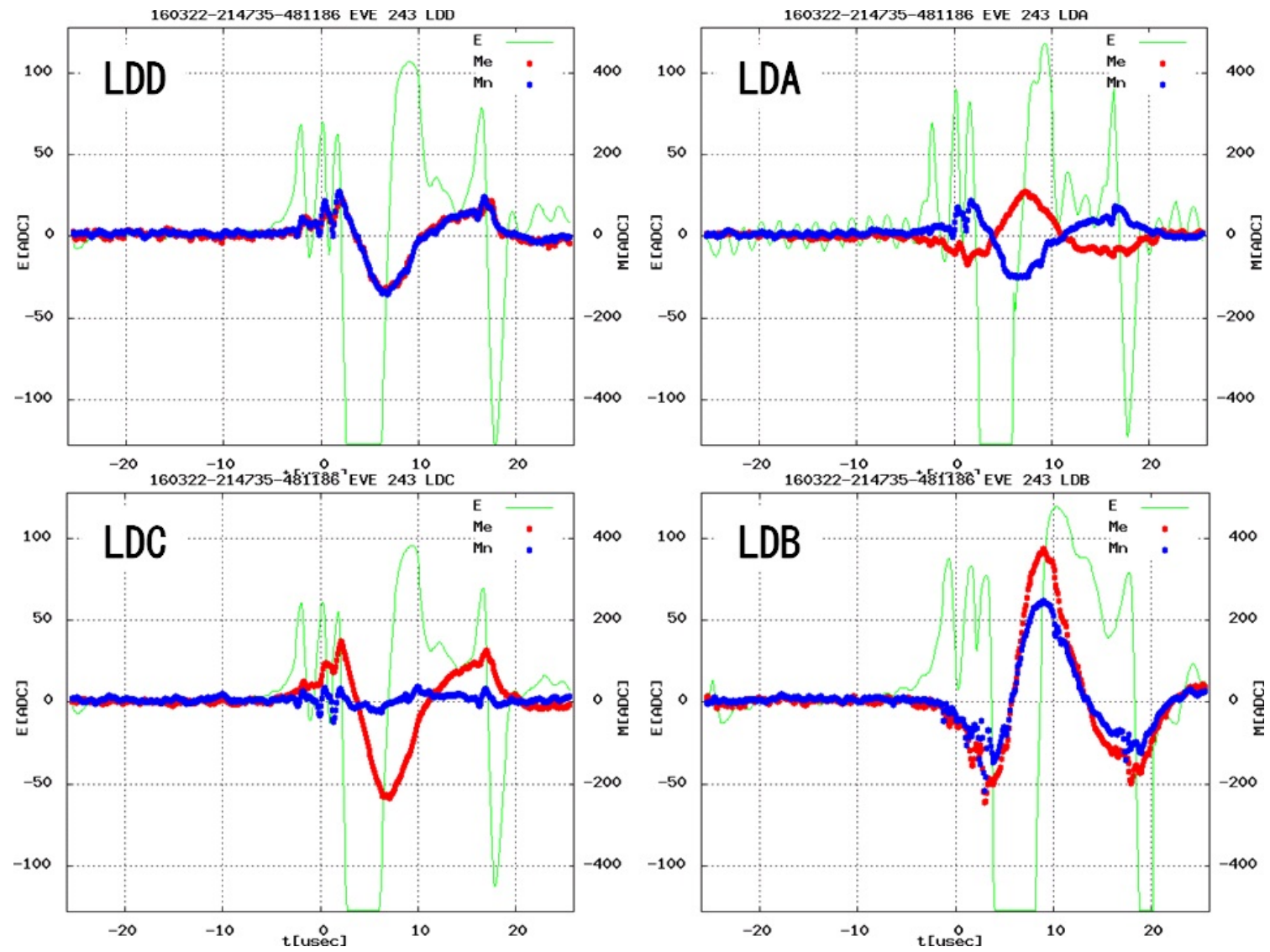

Figure 4: Waveforms for a 4-fold coincidence trigger. Green shows electric field. Red shows east-west magnetic field. Blue shows north-south magnetic field.

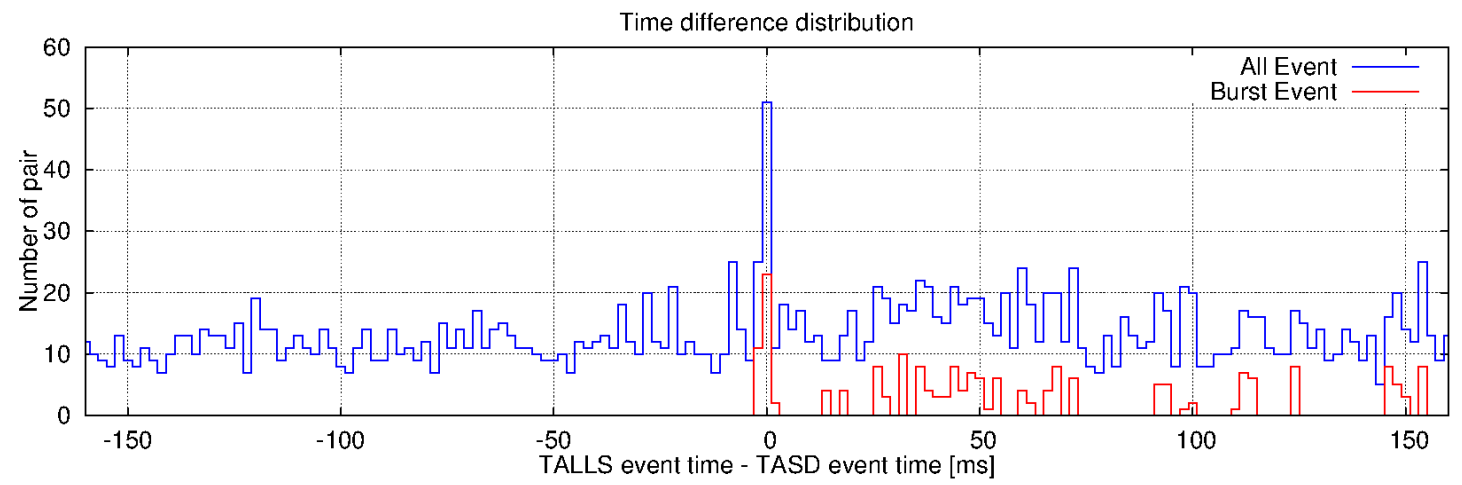

Figure 5: Time difference distribution between TASD and TALLS. The bin width is $2 \mathrm{~ms}$. The central bin shows TALLS event within $\pm 1 \mathrm{~ms}$ from TASD event. The left half shows the lightning discharge occurs prior to the air shower. 
antecedent. Figure $[$ and its feature completely reproduces the result shown in [ [ $]$, by different lightning detector.

\section{Plan and Summary}

The fifth detector(LDE) with solar battery system is ready to deploy to the central laser facility (Figure $\square$ ). However, the construction of expansion of TASD called TAx4 is going on now. There is possibility to deploy the fifth detector for the expanded area, so the location will be decided by near future condition.

MDF can be used to choose proper location in double root but has somewhat large error as written above. The unique location by 4-detectors TOA can be used to evaluate and calibrate MDF. Therefore, many lightning locations by TOA for a long time will be used for this purpose. And I will try to evaluate the peak current of lightning discharge by using amplitudes of the magnetic field waveform and the distances from the detector to the lightning location. Then, of course, I will check the correlation of lightning with TASD data.

Telescope Array Lightning Location System is running from September 2015 stably. The analysis for TALLS is going on.

\section{Acknowledgments}

TALLS is supported by the Mitsubishi Foundation and the joint research program of the Institute for Cosmic Ray Research, University of Tokyo.

The Telescope Array experiment is supported by the Japan Society for the Promotion of Science(JSPS) through Grants-in-Aid for Priority Area 431, for Specially Promoted Research JP21000002, for Scientific Research (S) JP19104006, for Specially Promoted Research JP15H05693, for Scientific Research (S) JP15H05741, for Science Research (A) JP18H03705 and for Young Scientists (A) JPH26707011; by the joint research program of the Institute for Cosmic Ray Research (ICRR), The University of Tokyo; by the U.S. National Science Foundation awards PHY-0601915, PHY-1404495, PHY-1404502, and PHY-1607727; by the National Research Foundation of Korea (2016R1A2B4014967, 2016R1A5A1013277, 2017K1A4A3015188, 2017R1A2A1A05071429) ; by the Russian Academy of Sciences, RFBR grant 16-02-00962a (INR), IISN project No. 4.4502.13, and Belgian Science Policy under IUAP VII/37 (ULB). The foundations of Dr. Ezekiel R. and Edna Wattis Dumke, Willard L. Eccles, and George S. and Dolores Doré Eccles all helped with generous donations. The State of Utah supported the project through its Economic Development Board, and the University of Utah through the Office of the Vice President for Research. The experimental site became available through the cooperation of the Utah School and Institutional Trust Lands Administration (SITLA), U.S. Bureau of Land Management (BLM), and the U.S. Air Force. We appreciate the assistance of the State of Utah and Fillmore offices of the BLM in crafting the Plan of Development for the site. Patrick Shea assisted the collaboration with valuable advice on a variety of topics. The people and the officials of Millard County, Utah have been a source of steadfast and warm support for our work which we greatly appreciate. We are indebted to the Millard County Road Department for their efforts to maintain and clear the roads which get us to our sites. 
We gratefully acknowledge the contribution from the technical staffs of our home institutions. An allocation of computer time from the Center for High Performance Computing at the University of Utah is gratefully acknowledged.

\section{References}

[1] T.Okuda, et al., PoS (2015), ICRC2015, 298

[2] R.U.Abbasi, et al., Phys. Lett. A (2017), 381, p2565-2572

[3] T.AbuZayyad, et al., NIM Phys. Res. A (2012), 689, p87-97

[4] C.B. Moore, et al., Geophys. Res. Lett. (2001), 28, p2141-2144.

[5] J.R. Dwyer, et al., Geophys. Res. Lett. (2005), 32 L01803.

[6] A.V. Gurevich, et al., Phys. Lett. A (2009), 373, p3550-3553. 